\title{
Association of Developmental Venous Anomalies with Demyelinating Lesions in Patients with Multiple Sclerosis
}

\author{
(DD.M. Rogers, DM.E. Peckham, (D) L.M. Shah, and (D)R.H. Wiggins III
}

\begin{abstract}
SUMMARY: We present 5 cases of demyelination in patients diagnosed with multiple sclerosis that are closely associated with a developmental venous anomaly. Although the presence of a central vein is a known phenomenon with multiple sclerosis plaques, demyelination occurring around developmental venous anomalies is an underreported phenomenon. Tumefactive demyelination can cause a diagnostic dilemma because of its overlapping imaging findings with central nervous system neoplasm. The relationship of a tumefactive plaque with a central vein can be diagnostically useful, and we suggest that if such a lesion is closely associated with a developmental venous anomaly, an inflammatory or demyelinating etiology should be a leading consideration.
\end{abstract}

ABBREVIATION: DVA = developmental venous anomaly

$\mathrm{T}$ he perivenular nature of demyelinating plaques in multiple sclerosis has been observed in many studies, with the earliest mention of this relationship found in an 1863 autopsy specimen. ${ }^{1,2}$ The central veins within demyelinating lesions are not usually perceived on conventional T2 and FLAIR MR imaging sequences, but inferred anatomically; a classic example of this is the demyelination occurring along callosal-septal medullary veins known as "Dawson fingers." Advances in MR techniques, such as 3T FLAIR ${ }^{\star}$, high-field 7T MR imaging, and susceptibility-weighted imaging, have helped to demonstrate that nearly all multiple sclerosis plaques are oriented along a central vein. ${ }^{3-5}$ This has recently led the North American Imaging in Multiple Sclerosis Cooperative to propose that the "central vein sign" has potential diagnostic utility to prospectively diagnose multiple sclerosis. ${ }^{6}$ Gaitán et $\mathrm{al}^{7}$ used dynamic contrast-enhanced 7T MR to show that smaller multiple sclerosis lesions enhance centrifugally, suggesting that these lesions grow outward from this central vein. ${ }^{7}$ Recently, Samaraweera et $\mathrm{al}^{8}$ showed that a central vein can be identified on clinical $3 \mathrm{~T}$ scanners by using $\mathrm{T}^{*}$ sequences with high echoplanar imaging.

Developmental venous anomalies (DVAs) have been found to be more prevalent in patients with multiple sclerosis. ${ }^{9}$ Despite this

Received May 20, 2017; accepted after revision July 12.

From the Department of Radiology and Imaging Science, University of Utah Medical Center, Salt Lake City, Utah.

Please address correspondence to Douglas M. Rogers, MD, 1861 South 1500 East, Salt Lake City, UT 84105; e-mail: Douglas.rogers@hsc.utah.edu

http://dx.doi.org/10.3174/ajnr.A5374 relationship, there is little research regarding the spatial proximity between DVAs and demyelinating plaques. A single published case report describes a focus of acute demyelination surrounding a DVA in the cerebellum, which was confirmed histologically. ${ }^{10}$ Strengthening this association may provide a useful clue in the diagnosis of tumefactive demyelination in the setting of confounding imaging findings.

We present 5 cases of demyelination in patients diagnosed with multiple sclerosis that are closely associated with a DVA.

\section{CASE SERIES}

\section{Case 1}

A 56-year-old woman with a medical history of hypertension and chronic lymphocytic (Hashimoto) thyroiditis initially presented with left-sided facial numbness, word-finding difficulty, and ataxia. Contrast-enhanced MR imaging demonstrated enhancing lesions within the left middle cerebellar peduncle and right cerebellum. The right cerebellar lesion was closely associated with a large DVA (Fig 1). Follow-up MR imaging 1 month later showed enlargement of these lesions, along with a new enhancing lesion in the left pons.

The initial differential diagnoses included acute demyelination and metastatic disease from an unknown primary malignancy. A CT of chest/abdomen/pelvis in search of a source of malignancy was negative, as was a lumbar puncture for malignant cells and oligoclonal bands. MR imaging perfusion examination demonstrated decreased $K^{\text {trans }}$, increased Ve, and decreased Vp within these lesions, consistent with increased permeability, decreased cellularity, and decreased vascularity, respectively. 


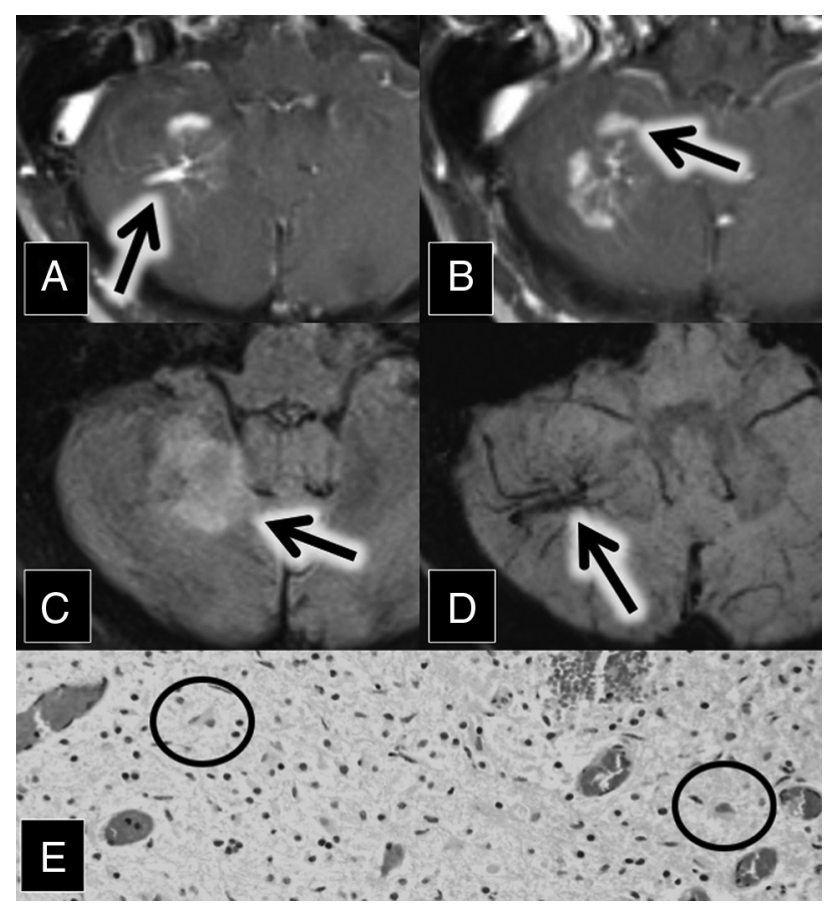

FIG 1. $A$ and $B$, Axial contrast-enhanced and fat-saturated MR imaging show a right cerebellar DVA ( $A$, arrow) surrounded by patchy parenchymal enhancement ( $B$, arrow). $C$, Axial FLAIR MR imaging shows corresponding hyperintense lesion in the right cerebellum (arrow). D, Axial susceptibility-weighted imaging maximum intensity projection shows a large right cerebellar DVA (arrow). E, H\&E staining $20 \times$ shows moderately hypercellular cerebellar white matter with infiltrating macrophages (circled). In addition, immunohistochemical staining demonstrated intact neurofilament and positive CD68 histiocytes, and Luxol fast blue stain showed myelin loss (not pictured).

Taken together, the findings were consistent with acute demyelination, and this was dictated and verbally reported to the referring neurosurgeons. Persistent clinical uncertainty led to an excisional biopsy of the right cerebellar lesion. Demyelination was histologically confirmed (Fig 1). Imaging performed 3 months postoperatively demonstrated decreased size and enhancement of the pontine and left middle cerebellar peduncle lesions. A year later, her facial numbness and ataxia resolved, and she subsequently developed increased fatigue and lack of concentration. Based on the imaging and the pathologic findings, the patient was diagnosed by her neurologist with multiple sclerosis and has not had a relapse while on fingolimod therapy.

\section{Case 2}

A 37-year-old man with a medical history of transverse myelitis presented with gradually increasing blurry vision of his left eye, which was mildly painful. MR imaging and ophthalmologic examination were consistent with left optic neuritis (Fig 2).

After treatment with steroids, the patient improved. He did not tolerate treatment with natalizumab because of an allergic reaction, so he was started on dimethyl fumarate. He returned 15 months later with left homonymous hemianopsia and right visual field constriction. MR imaging at this time demonstrated left frontal, left parietal, and right temporal lesions with

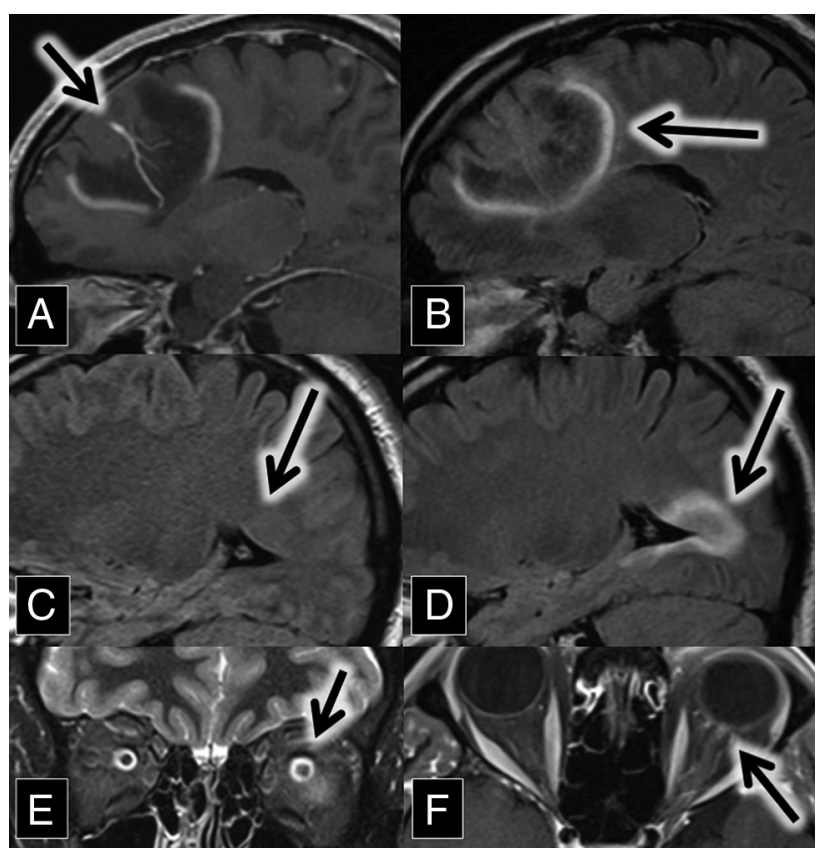

FIG 2. $A$ and $B$, Sagittal contrast-enhanced $T 1$ and FLAIR MR images show a large left frontal lobe DVA ( $A$, arrow) surrounded by a lesion with discontinuous leading-edge enhancement ( $B$, arrow), typical for tumefactive demyelination. $C$ and $D$, Sagittal FLAIR MR images at the same anatomic location with a 15-month interval show the development of a demyelinating plaque adjacent to the occipital horn of the right lateral ventricle (arrows). E, Coronal T2 with fat-saturation MR imaging shows left optic nerve hyperintensity (arrow). The patient presented with optic neuritis 15 months before imaging in $A$ and $B$. F. Axial contrast-enhanced Tl with fat saturation MR imaging shows left optic nerve enhancement (arrow) in the setting of optic neuritis.

discontinuous leading-edge enhancement, typical for acute tumefactive demyelination. Postcontrast images demonstrated that the left frontal lobe lesion was centered on a large DVA (Fig 2). Spectroscopy was obtained, which showed elevated choline and decreased $\mathrm{N}$-acetyl aspartate, suggestive of active cell membrane turnover with underlying neuronal loss.

The patient was treated with methylprednisolone, leading to improvement of his vision to near baseline. Given the pathognomonic brain lesions with peripheral discontinuous enhancement, along with the preceding history of optic neuritis and transverse myelitis, a neurologist diagnosed the patient with relapsing-remitting multiple sclerosis.

\section{Case 3}

A 46-year-old man with a history of relapsing-remitting multiple sclerosis, renal stones, and gastric ulcers related to Helicobacter pylori infection presented for routine follow-up. MR imaging from 2 years prior demonstrated multiple white matter and spinal cord FLAIR hyperintense lesions, predominantly in a callosal and pericallosal distribution typical of multiple sclerosis. A new enhancing lesion was present in the left middle cerebellar peduncle, closely associated with a left cerebellar DVA (Fig 3). The T2 sequence demonstrated the central vein sign. The enhancement of this lesion was no longer present on the follow-up MR imaging 2 years later.

The patient had multiple relapses over several years, with tran- 
sient symptoms including vertical diplopia, ataxia, and, most recently, left-sided extremity numbness. Despite this, he remains physically active. He continues to be followed by a neurologist and has avoided an exacerbation over the past 5 years on dimethyl fumarate therapy.

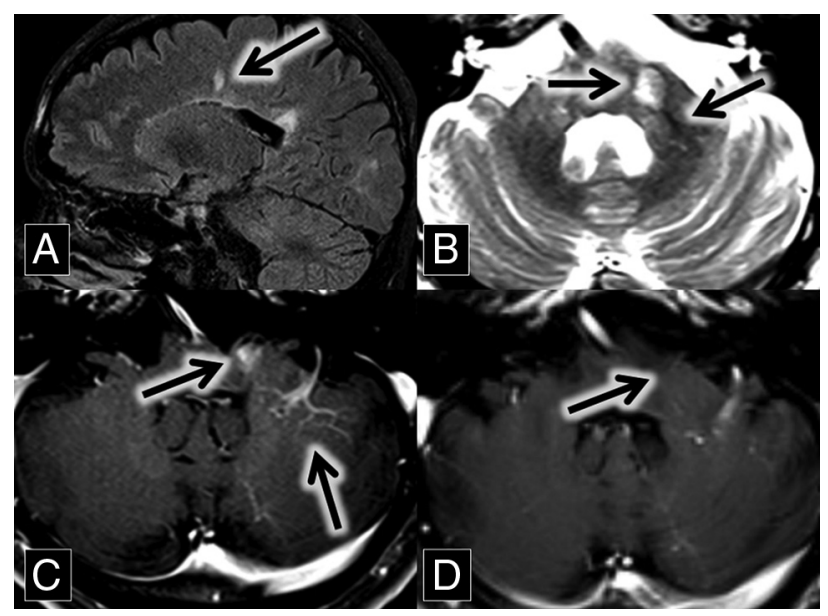

FIG 3. A, Sagittal FLAIR MR imaging shows multiple hyperintense callosal and pericallosal multiple sclerosis plaques (Dawson fingers; arrow) B, Axial T2 MR imaging shows a hyperintense left middle cerebellar peduncle lesion (midline arrow) associated with a flow void (central vein sign; left lateral arrow), which extends from the DVA better demonstrated in image $C$. $C$ and $D$, Axial contrast-enhanced $T 1$ MR images show an enhancing left middle cerebellar peduncle lesion (midline arrow) closely associated with a left cerebellar DVA (left lateral arrow). This enhancement was resolved on the follow-up scan at the same anatomic level 2 years later ( $D$, arrow).

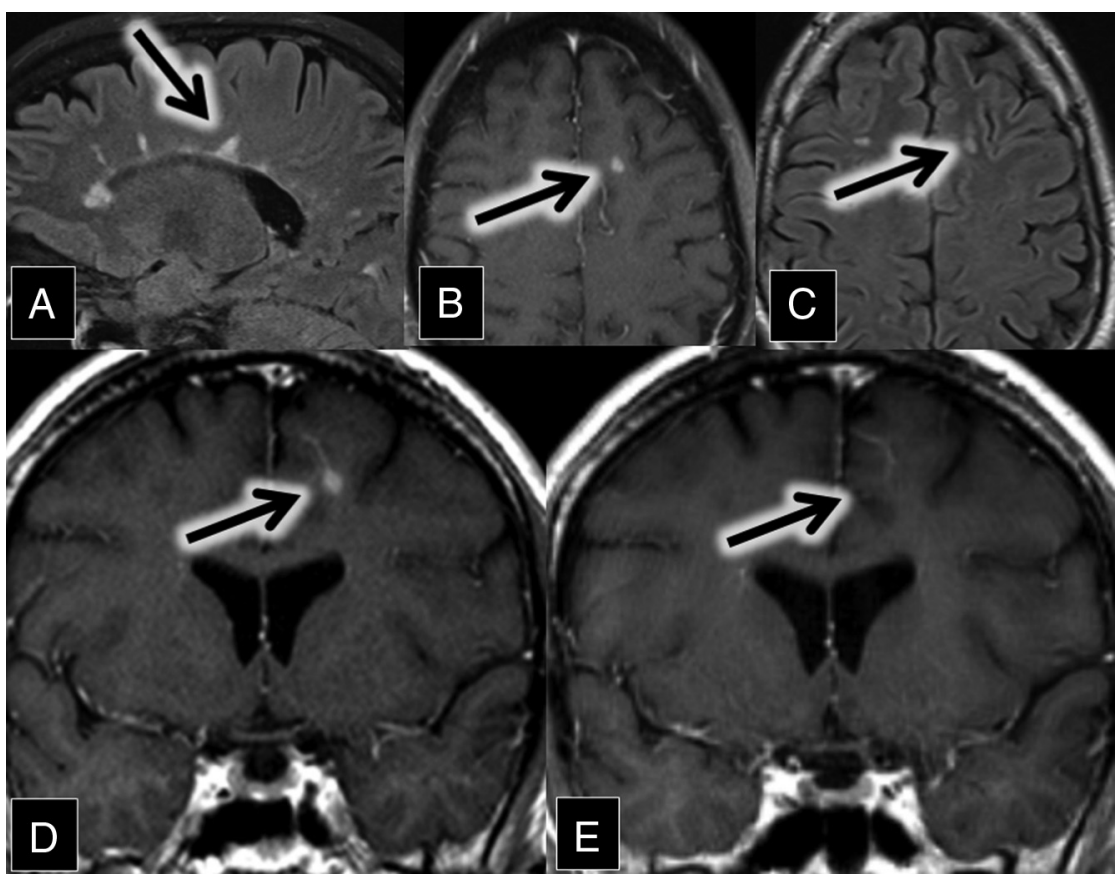

FIG 4. A, Sagittal FLAIR MR imaging shows multiple hyperintense callosal and pericallosal multiple sclerosis plaques (consistent with Dawson fingers; arrow). B. Axial contrast-enhanced $T 1$ and $C$, FLAIR MR images show a FLAIR hyperintense ( $C$, arrow) and enhancing ( $B$, arrow) lesion in the left frontal lobe. $D$ and $E$, Coronal contrast-enhanced T1 MR images show an enhancing left frontal lobe lesion ( $D$, arrow) closely associated with a DVA. This enhancement was resolved on the follow-up scan 9 months later ( $E$, arrow).

\section{Case 4}

A 47-year-old man with relapsing-remitting multiple sclerosis presented for evaluation of new diplopia secondary to internuclear ophthalmoplegia. MR imaging demonstrated numerous white matter and spinal cord FLAIR and T2 hyperintense lesions in a pattern typical of multiple sclerosis. A review of his imaging from 7 years prior showed an interim development of a new enhancing demyelinating lesion closely associated with a left frontal lobe DVA (Fig 4). The enhancement resolved on the follow-up MR imaging 9 months later.

A neurologist initially diagnosed him with multiple sclerosis in his early 20s after recurrent episodes of optic neuritis. Since then, he had multiple relapses, with symptoms dominated by progressive gait difficulty and spasticity, neuropathic pain, and depression. The patient continues to be followed by a neurologist and is currently being treated with rituximab.

\section{Case 5}

A 27-year-old woman with relapsing-remitting multiple sclerosis presented for routine follow-up. MR imaging demonstrated multiple subcortical and periventricular FLAIR hyperintense lesions, including callosal and pericallosal plaques typical of multiple sclerosis. A comparison of axial FLAIR and T1 contrast-enhanced images demonstrated a grouping of FLAIR hyperintense lesions around a right parietal DVA. These lesions were new compared with MR imaging from 8 months prior, most consistent with new demyelinating lesions (Fig 5).

The patient had no motor or sensory deficits, but she struggled with symptoms of chronic fatigue and cognitive dysfunction, with occasional word-finding difficulty. She continues to be followed by a neurologist and treated with maintenance therapy of interferon $\beta$-1a.

\section{DISCUSSION}

The relationship of demyelination and DVAs is underreported, despite the presence of a central vein as a known phenomenon with multiple sclerosis plaques. This may be due in part to limitations of conventional imaging because both abnormalities may not be apparent together on the same standard MR imaging sequence. DVAs are easily seen on contrast-enhanced images, whereas multiple sclerosis plaques are best seen on T2 and FLAIR sequences. Occasionally, demyelination is enhancing in its acute phase, allowing the association with a DVA to be demonstrated on contrast-enhanced images (as shown in cases 1-4). Sometimes, a DVA may be large enough that the flow void is clearly identified within the plaque on a T2 sequence. Although advanced MR imaging sequences such as 3 T FLAIR ${ }^{*}$ and high-field 7T MR imaging can improve the detection of the central vein in demyelinating plaques, the central vein 


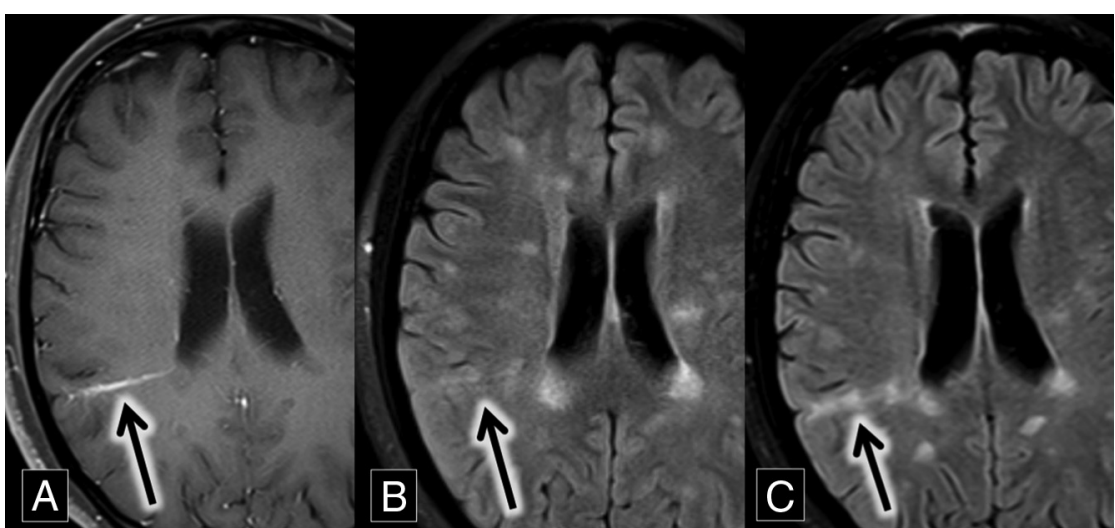

FIG 5. A, Axial contrast-enhanced TIMR imaging shows a right parietal lobe DVA (arrow). B and C, Axial FLAIR MR images over an 8-month interval show development of right parietal lobe hyperintense plaques ( $B$ and $C$, arrows) oriented along the course of the DVA demonstrated in $A$. a DVA. Further investigation is required to determine whether the demonstrated parenchymal abnormalities around DVAs increase the propensity to develop demyelination around these typically benign vascular lesions in the setting of multiple sclerosis.

\section{CONCLUSIONS}

Demyelination surrounding DVAs is an underreported and potentially dangerously unrecognized phenomenon. Tumefactive demyelination may be difficult to differentiate from central nervous system neoplasm on imaging, and an association of the demyelinating lesion with a DVA should instigate further can also be identified on clinical $3 \mathrm{~T}$ scanners by using $\mathrm{T} 2{ }^{\star}$ sequences with high echo-planar imaging. ${ }^{8}$ It should be acknowledged that although MR imaging is a useful tool in expediting the diagnosis of multiple sclerosis by demonstrating the dissemination of lesions in space and time, clinical criteria remain the criterion standard for diagnosis. ${ }^{11}$

Tumefactive demyelination can cause a diagnostic dilemma because of its overlapping imaging findings with central nervous system neoplasm. The relationship of a tumefactive plaque with a central vein can be diagnostically useful,,$^{3-6,8,12-15}$ and we suggest that if such a lesion is closely associated with a DVA, an inflammatory or demyelinating etiology should be a leading consideration. In addition to correlation with other clinical and imaging clues, further evaluation with MR imaging perfusion and MR imaging spectroscopy can also aid in the diagnosis in such cases, distinguishing neoplastic from non-neoplastic lesions, thereby avoiding biopsy. ${ }^{16}$ Biopsy in the presence of a DVA will carry an additional risk of infarction and/or hemorrhage beyond the surgical margin because the DVA may represent the sole venous outflow to adjacent normal brain parenchyma. ${ }^{17-19}$ Another noninvasive strategy is short-term imaging follow-up because the enhancement of a demyelinating lesion should decrease and/or resolve over time.

The predisposition for demyelination to occur around normally distributed cerebral veins is well-known, ${ }^{6}$ and we hypothesize that the medusa-like DVA may actually create an even more compelling milieu for this process to occur. Once thought to be benign and incidental findings, recent studies show that brain tissue surrounding DVAs is abnormal in most cases. A comparison of MR imaging and PET in patients with DVAs demonstrated surrounding hypometabolism in $75 \%$ of cases. ${ }^{20}$ On MR imaging perfusion, MTT and TTP are prolonged around most DVAs, suggestive of venous congestion in these regions. ${ }^{21}$

The pathophysiology and histopathology of multiple sclerosis plaques is characterized by lymphocytes and monocytes crossing the blood-brain barrier via venous channels, causing perivenular cuffing in active lesions. ${ }^{22,23}$ These lymphocytes interact with autoantigens such as myelin basic protein, leading to a local inflammatory response and subsequent demyelination. ${ }^{6,24,25}$ We hypothesize that the rate and/or amount of lymphocytic infiltration may be accelerated in the setting of impaired venous drainage by noninvasive testing such as MR perfusion imaging in an attempt to avoid biopsy and possible associated complications. The presented cases support the perivenular nature of multiple sclerosis.

\section{REFERENCES}

1. Rindfleisch E. Histologisches Detail zu der grauen Degeneration von Gehirn und Rückenmark. (Zugleich ein Beitrag zu der Lehre von der Entstehung und Verwandlung der Zelle.) Archiv für pathologische Anatomie und Physiologie und für klinische Medicin 1863;26: 474-83 CrossRef

2. Rae-Grant AD, Wong C, Bernatowicz R, et al. Observations on the brain vasculature in multiple sclerosis: a historical perspective. Mult Scler Relat Disord 2014;3:156-62 CrossRef Medline

3. Solomon AJ, Schindler MK, Howard DB, et al. "Central vessel sign” on 3T FLAIR ${ }^{\star}$ MRI for the differentiation of multiple sclerosis from migraine. Ann Clin Transl Neurol 2016;3:82-87 CrossRef Medline

4. Mistry N, Abdel-Fahim R, Samaraweera A, et al. Imaging central veins in brain lesions with 3 - $\mathrm{T}$ T $2 *$-weighted magnetic resonance imaging differentiates multiple sclerosis from microangiopathic brain lesions. Mult Scler 2016;22:1289-96 CrossRef Medline

5. Tallantyre EC, Brookes MJ, Dixon JE, et al. Demonstrating the perivascular distribution of MS lesions in vivo with 7-Tesla MRI. Neurology 2008;70:2076-78 CrossRef Medline

6. Sati P, Oh J, Constable RT, et al. The central vein sign and its clinical evaluation for the diagnosis of multiple sclerosis: a consensus statement from the North American Imaging in Multiple Sclerosis Cooperative. Nat Rev Neurol 2016;12:714-22 CrossRef Medline

7. Gaitán MI, Sati P, Inati SJ, et al. Initial investigation of the bloodbrain barrier in MS lesions at 7 Tesla. Mult Scler 2013;19:1068-73 CrossRef Medline

8. Samaraweera AP, Clarke MA, Whitehead A, et al. The central vein sign in multiple sclerosis lesions is present irrespective of the $\mathrm{T}^{*}{ }^{*}$ sequence at 3 T. J Neuroimaging 2017;27:114-21 CrossRef Medline

9. Zhao G, Li DK, Traboulsee T, et al. Developmental venous anomalies (venous angiomas) on MRI are more common in patients with multiple sclerosis. In: Proceedings of the International Society for Magnetic Resonance in Medicine Twelfth Scientific Meeting, Kyoto, Japan. May 15-21, 2004

10. Jung G, Schroder R, Lanfermann H, et al. Evidence of acute demyelination around a developmental venous anomaly: magnetic resonance imaging findings. Invest Radiol 1997;32:575-77 CrossRef Medline

11. Filippi M, Rocca MA, Ciccarelli O, et al. MRI criteria for the diagnosis of multiple sclerosis: MAGNIMS consensus guidelines. Lancet Neurol 2016;15:292-303 CrossRef Medline

12. Lane JI, Bolster B, Campeau NG, et al. Characterization of multiple sclerosis plaques using susceptibility-weighted imaging at $1.5 \mathrm{~T}$ : 
can perivenular localization improve specificity of imaging criteria? J Comput Assist Tomogr 2015;39:317-20 CrossRef Medline

13. Zhang Y, Metz LM. Dominant perivenular enhancement of tumefactive demyelinating lesions in multiple sclerosis. Neurology 2010; 75:1396 CrossRef Medline

14. Tan IL, van Schijndel RA, Pouwels PJ, et al. MR venography of multiple sclerosis. AJNR Am J Neuroradiol 2000;21:1039-42 Medline

15. Dal-Bianco A, Hametner S, Grabner G, et al. Veins in plaques of multiple sclerosis patients-a longitudinal magnetic resonance imaging study at 7 Tesla. Eur Radiol 2015;25:2913-20 CrossRef Medline

16. Hourani R, Brant LJ, Rizk T, et al. Can proton MR spectroscopic and perfusion imaging differentiate between neoplastic and nonneoplastic brain lesions in adults? AJNR Am J Neuroradiol 2008;29: 366-72 CrossRef Medline

17. Nagatani K, Osada H, Takeuchi S, et al. Surgical resection of developmental venous anomaly causing massive intracerebral haemorrhage: a case report. Br J Neurosurg 2014;28:116-18 CrossRef Medline

18. Amuluru K, Al-Mufti F, Hannaford S, et al. Symptomatic infratentorial thrombosed developmental venous anomaly: case report and review of the literature. Interv Neurol 2016;4:130-37 CrossRef Medline
19. Rammos SK, Maina R, Lanzino G. Developmental venous anomalies: current concepts and implications for management. Neurosurgery 2009;65:20-29; discussion 29-30 CrossRef Medline

20. Larvie M, Timerman D, Thum JA. Brain metabolic abnormalities associated with developmental venous anomalies. AJNR Am J Neuroradiol 2015;36:475-80 CrossRef Medline

21. Jung HN, Kim ST, Cha J, et al. Diffusion and perfusion MRI findings of the signal-intensity abnormalities of brain associated with developmental venous anomaly. AJNR Am J Neuroradiol 2014;35: 1539-42 CrossRef Medline

22. Matthews PM, Roncaroli F, Waldman A, et al. A practical review of the neuropathology and neuroimaging of multiple sclerosis. Pract Neurol 2016;16:279-87 CrossRef Medline

23. Tanaka R, Iwasaki Y, Koprowski H. Ultrastructural studies of perivascular cuffing cells in multiple sclerosis brain. Am J Pathol 1975;81:467-78 Medline

24. Adams CW. The onset and progression of the lesion in multiple sclerosis. J Neurol Sci 1975;25:165-82 CrossRef Medline

25. Minagar A, Alexander JS. Blood-brain barrier disruption in multiple sclerosis. Mult Scler 2003;9:540 - 49 CrossRef Medline 